\title{
Pulmonary Endothelial Impairment During Gefitinib Therapy: A Preliminary Assessment with Iodine-123-Metaiodobenzylguanidine (123I- MIBG) Scintigraphy
}

\author{
Tomonobu Koizumi*, Kazuhisa Urushihata, Tadashige Fujii and Keishi Kubo \\ First Department of Internal Medicine, Shinshu University School of Medicine, 3-1-1 Asahi Matsumoto, 390-8621, \\ Japan
}

\begin{abstract}
Iodine-123-metaiodobenzylguanidine $\left({ }^{123} \mathrm{I}-\mathrm{MIBG}\right)$ kinetics in the lung could serve as a novel diagnostic tool to evaluate endothelial damage. Interstitial lung disease (ILD) associated with gefitinib, an epidermal growth factor receptor (EGFR)-tyrosine kinase inhibitor (TKI), has been reported as a serious adverse effect. This study was performed to examine the possibility that gefitinib induces pulmonary endothelial damage. Serial ${ }^{123}$ I-MIBG scintigraphy was performed in 5 patients with non-small cell lung cancer before and one month after initiation of gefitinib treatment. Anterior planar images were acquired $15 \mathrm{~min}$ after injection of ${ }^{123} \mathrm{I}-\mathrm{MIBG}$ and the total lung to upper mediastinum ratio $(\mathrm{L} / \mathrm{M})$ was calculated in both lungs. None of the patients developed ILD during the study. There were no significant differences in the values of $\mathrm{L} / \mathrm{M}$ before and after gefitinib therapy. These findings suggest that gefitinib has little influence on the pulmonary endothelium in patients with no signs of ILD.
\end{abstract}

Keywords: Interstitial lung disease, acute lung injury, non-small cell lung cancer, EGFR, drug-induced lung injury.

\section{INTRODUCTION}

Iodine-123-metaiodobenzylguanidine $\left({ }^{123} \mathrm{I}-\mathrm{MIBG}\right)$ has been widely used for the detection of various neuroendocrine tumors and evaluation of adrenergic dysfunction in the heart $[1,2] .{ }^{123}$ I-MIBG is taken up by the lung through a saturable, energy-requiring, sodium-dependent transport mechanism similar to biogenic amines, such as serotonin and norepinephrine $[3,4]$. It is well known that transport of these biogenic amines requires normal endothelial cell integrity. Thus, ${ }^{123}$ I-MIBG is regarded as an indicator of pulmonary endothelial function [3-6], because it behaves in a quantitatively similarly manner to norepinephrine in the pulmonary circulation [4]. Slosman et al. initially evaluated lung uptake of ${ }^{123}$ I-MIBG using animal models and demonstrated decreased ${ }^{123}$ I-MIBG lung extraction in bleomycin-induced endothelial injury [6]. Furthermore, several clinical studies using this property of ${ }^{123}$ I-MIBG scintigraphy showed that decreased ${ }^{123}$ I-MIBG kinetics in the lung could serve as a novel diagnostic tool to evaluate the endothelial damage in patients with pulmonary fibrosis [7], myeloperoxidase anti-neutrophil cytoplasmic antibodypositive vasculitis [8], high altitude-related hypoxia [9], and high altitude pulmonary edema [10].

Gefitinib is an epidermal growth factor receptor (EGFR)tyrosine kinase inhibitor (TKI) and shows antitumor activity in patients with advanced non-small cell lung cancer (NSCLC) [11]. In particular, higher response rates have been reported in Asian EGFR mutation-positive NSCLC patients

*Address correspondence to this author at the The First Department of Internal Medicine, Shinshu University School of Medicine, 3-1-1 Asahi Matsumoto, 390-8621, Japan; Tel: +81-263-37-2631; Fax: +81-263-363722; E-mail: tomonobu@shinshu-u.ac.jp
$[12,13]$. However, cases of fatal gefitinib-induced interstitial lung disease (ILD) have been reported [14-16], and higher incidence rates of ILD have been reported in Japanese NSCLC patients receiving gefitinib than in other countries $[15,16]$. The underlying mechanisms of gefitinib-induced ILD remain unclear. EGFR is expressed on a number of cells of the lungs, including epithelial cells, smooth muscle cells, fibroblasts, and endothelial cells [17, 18]. Gefitinib-induced ILD shows diffuse alveolar damage, including epithelial and endothelial areas.

Our hypothesis was that gefitinib caused pulmonary endothelial damage in patients with NSCLC, even in those with no clinical respiratory manifestations and/or abnormal chest radiographic findings. Thus, the present study was performed to determine the effects of gefitinib administration on the pulmonary uptake of ${ }^{123}$ I-MIBG before and after gefitinib therapy.

\section{MATERIALS AND METHODS}

This study conformed with the provisions of the Declaration of Helsinki 1995. All subjects were informed of the procedures and risks of this study, and informed consent was obtained prior to participation in the study.

\section{Subjects}

Patients with histologically or cytologically confirmed NSCLC were enrolled in this study. Patients with active infection, interstitial pneumonia on chest computed tomography (CT), pericardial effusion that required drainage, active brain metastasis, pregnancy, or Eastern Clinical Oncology Group (ECOG) performance status $>2$ were excluded from the trial. In addition, patients who showed atelectasis due to tumor mass or pleural effusion on chest radiograph were also excluded. Other concomitant 
Table 1. The Clinical Characteristics of Patients Enrolled in this Study and the ${ }^{123}$ I-MIBG Lung Uptake (L/M)

\begin{tabular}{|c|c|c|c|c|c|c|c|c|}
\hline \multirow[b]{2}{*}{ Case } & \multirow[b]{2}{*}{ Age } & \multirow[b]{2}{*}{ Gender } & \multirow{2}{*}{$\begin{array}{c}\text { Smoking } \\
\text { (Pack } \cdot \text { yrs) }\end{array}$} & \multirow[b]{2}{*}{ Histology } & \multirow{2}{*}{$\begin{array}{c}\text { Previous } \\
\text { Chemotherapy } \\
\text { (current Line) }\end{array}$} & \multirow{2}{*}{$\begin{array}{l}\text { Response } \\
\text { to Gefitinib }\end{array}$} & \multicolumn{2}{|c|}{ (rt.L/M+lt.L/M)/2 } \\
\hline & & & & & & & $\begin{array}{c}\text { Before } \\
\text { Treatment }\end{array}$ & $\begin{array}{c}\text { After } \\
1 \text { Month }\end{array}$ \\
\hline 1 & 61 & M & 82 & $\mathrm{Sq}$ & $+(4 \mathrm{th})$ & SD & 1.33 & 1.35 \\
\hline 2 & 73 & M & 106 & $\mathrm{Sq}$ & $-(1 \mathrm{st})$ & $\mathrm{SD}$ & 1.26 & 1.36 \\
\hline 3 & 47 & $\mathrm{~F}$ & 12 & $\mathrm{Ad}$ & $+(3 \mathrm{rd})$ & SD & 1.61 & 1.82 \\
\hline 4 & 79 & M & 0 & Ad & $+(3 \mathrm{rd})$ & $\mathrm{SD}$ & 1.19 & 1.26 \\
\hline 5 & 69 & $\mathrm{~F}$ & 0 & $\mathrm{Ad}$ & $+(4$ th $)$ & SD & 1.77 & 1.78 \\
\hline average & 65.0 & & & & & & 1.35 & 1.44 \\
\hline$\pm \mathrm{SD}$ & 14.1 & & & & & & 0.18 & 0.25 \\
\hline
\end{tabular}

anticancer therapy or experimental drug administration of any type was not permitted. Furthermore, none of the subjects were receiving medications that could affect ${ }^{123} \mathrm{I}-$ MIBG uptake and metabolism, such as beta-adrenergic blocking drugs, catecholamine, and angiotensin converting enzyme inhibitors.

${ }^{123}$ I-MIBG scintigraphy was performed before and one month after the initiation of gefitinib therapy. Patients were started on treatment with gefitinib at a dose of $250 \mathrm{mg}$ daily after initial ${ }^{123}$ I-MIBG scintigraphy. Patients were monitored by chest radiography and blood gas analysis during gefitinib therapy. If the enrolled patient showed a good response and achieved partial response assessed by the response evaluation criteria in solid tumors (RECIST Version 1.0) within one month after commencement of gefitinib therapy, second examination of ${ }^{123}$ I-MIBG scintigraphy was canceled, because we speculated that radiographic improvement could affect the ${ }^{123}$ I-MIBG lung uptake.

\section{MIBG Scintigraphy}

A dose of $111 \mathrm{MBq}$ of ${ }^{123}$ I-MIBG (Daiichi Radioisotopes Labs, Tokyo, Japan) was administered intravenously after a 15-min rest period with the patient lying undisturbed in bed. Anterior planar images were acquired 15 min after injection of ${ }^{123} \mathrm{I}-\mathrm{MIBG}$, and stored in a $64 \times 64$ matrix by a scintillation camera (ZLC 7500; Siemens, Solna, Sweden) equipped with a low-energy, general purpose collimator interfaced to a minicomputer (SCINTIPAC 2400; Shimadzu, Kyoto, Japan). The energy window was set at the $159 \mathrm{keV}$ photopeak of ${ }^{123} \mathrm{I}$. The region of interest (ROI) was placed over the upper mediastinum, the right and left lung in planar images. ROIs corresponding to the contours of the right and left lungs were manually assigned with reference to the isocount line. Total counts of each lung and heart were measured and the geometric mean was calculated as counts per pixel. To quantify the degree of lung uptake of ${ }^{123} \mathrm{I}-\mathrm{MIBG}$, the lung to upper mediastinum ratio in ${ }^{123} \mathrm{I}-\mathrm{MIBG}$ uptake (L/M) was measured for the right (R) and left (L) lungs. These data were used to calculate the mean value $(\mathrm{R}+\mathrm{L} / 2 ; \mathrm{L} / \mathrm{M})$.

\section{Statistical Analysis}

Data are expressed as means \pm SD. The paired $t$ test was used to analyze differences in ${ }^{123}$ I-MIBG scintigraphic data. In all analyses, $P<0.05$ was taken to indicate statistical significance.

\section{RESULTS}

In the present study, total nine patients with NSCLC (5 men and 4 women, with a mean age of 61.0 years, range 34 79 years) were enrolled in the present study. Among them, 4 subjects showed good response to gefitinib within one month and excluded from the following examination of ${ }^{123} \mathrm{I}-\mathrm{MIBG}$ lung uptake. Thus, the comparative analysis of serial ${ }^{123} \mathrm{I}-$ MIBG lung uptake was performed using 5 patients. The clinical characteristics and the ${ }^{123}$ I-MIBG lung uptake (L/M) in the present study are summarized in Table 1 . There were three men and two women, with a mean age of 65.0 years, range $47-79$ years. Three patients were ex-smokers. The histological types were squamous cell carcinoma (2 cases) and adenocarcinoma (3 cases). The response in all patients within one month was stable disease. The value of $\mathrm{L} / \mathrm{M}$ in nine enrolled patients before gefitinib therapy was $1.44 \pm$ 0.25 , which was not statistically significant from that in selected 5 subjects for comparison with following ${ }^{123}$ I-MIBG examination. In the 5 subjects, there were no statistically significant differences in ${ }^{123}$ I-MIBG lung uptake before $(1.35$ \pm 0.18 , ranging from 1.19 to 1.77$)$ and after $(1.44 \pm 0.25$, ranging from 1.26 to 1.82 ) gefitinib treatment.

\section{DISCUSSION}

In this study, serial evaluations of ${ }^{123}$ I-MIBG lung uptake were performed in patients with NSCLC before and after gefitinib treatment. None of the subjects developed ILD or showed new abnormal radiographic findings in the lungs. There were no statistically significant differences in ${ }^{123} \mathrm{I}-$ MIBG lung uptake before and after gefitinib treatment, suggesting that gefitinib administration did not cause pulmonary endothelial damage.

In terms of gefitinib-induced ILD, the interaction between epithelial cells or fibroblasts and the expression of EGFR has been discussed [19,20]. Indeed, the levels of epithelial expression of EGFR and its ligands, EGF and transforming growth factor- $\alpha$, were increased in fibrotic lung disease [19]. However, the overexpression of EGFR in tumor-associated endothelial cells from clinical specimens of human lung cancer has also been reported [18]. The appearance of ILD in patients treated with gefitinib was 
uncommonly rapid and the pathological finding was diffuse alveolar damage, similar to acute lung injury. The pathophysiology mainly involved damage to both epithelial and endothelial cells. EGFR-TKI could affect biological status of pulmonary endothelial cells, including apoptosis [18]. Therefore, in the present study, we focused on whether gefitinib could influence pulmonary endothelial cell impairment in a clinical situation using ${ }^{123}$ I-MIBG lung uptake. The onset of gefitinib-induced ILD is frequency observed within one month $[15,16]$. Therefore, second examination of ${ }^{123}$ I-MIBG lung uptake in the present study was selected at one month after the start of gefitinib therapy.

There were limitations to interpretation of our results in the present study. First, in contrast to our hypothesis, we found that gefitinib itself does not directly influence endothelial cells and that gefitinib-induced ILD is dependent on the individual. That is, pulmonary endothelial damage may be induced secondary to the development of gefitinibinduced ILD. Indeed, animal experiments showed no evidence that gefitinib induces lung injury in intact rat lungs [20]. Second, the number of patients enrolled in this study was too small to detect endothelial damage by ${ }^{123} \mathrm{I}-\mathrm{MIBG}$ lung uptake. We have demonstrated that only 6 cases of myeloperoxidase anti-neutrophil cytoplasmic antibodypositive vasculitis were enough to detect the impaired pulmonary endothelial function [8]. However, Takabatake et al. [7] summarized ${ }^{123}$ I-MIBG lung uptake in 23 patients with idiopathic pulmonary fibrosis and demonstrated significantly decreased ${ }^{123}$ I-MIBG lung uptake in patients with idiopathic pulmonary fibrosis compared with controls. They concluded that endothelial cell injury played a significant role in the pathogenesis of idiopathic pulmonary fibrosis. Based on these studies, further case studies might be needed to draw definitive conclusions.

Risk factors for gefitinib-induced ILD have been identified in a number of studies in the Japanese population, including sex, history of smoking, concurrent interstitial pneumonitis, history of prior chemotherapy, and poor performance status $[15,16]$. However, little information is available regarding predictive factors for gefitinib-induced ILD. Thus, it is necessary to find markers to elucidate which patients are at high risk of ILD before gefitinib therapy. The present study failed to demonstrate the usefulness of ${ }^{123} \mathrm{I}$ MIBG lung uptake. However, we believe that pulmonary endothelial impairment due to exposure to the administered drug could be involved. Determination of the scintigraphic kinetic behavior of ${ }^{123}$ I-MIBG in the lung may yield some new insight for detecting pulmonary endothelial damage.

\section{CONFLICT OF INTEREST}

None

\section{REFERENCES}

[1] Sisson JC, Wieland DM, Sherman P, Mangner TJ, Tobes MC, Jacques S Jr. Metaiodobenzylguanine as an index of the adrenergic nervous system integrity and function. J Nucl Med 1987; 28: 16204.

[2] Schofer J, Spielmann R, Schuchert A, Weber K, Schluter M. Iodine-123-Metaiodobenzyguanine scintigraphy: a noninvasive method to demonstrate myocardial adrenergic nervous system disintegrity in patients with idiopathic dilated cardiomyopathy. J Am Coll Cardiol 1988; 12: 1252-8.

[3] Slosman D, Morel D, Alderson P. A new imaging approach to quantitative evaluation of pulmonary vascular endothelial metabolism. J Thorac Imag 1988; 31: 49-52.

[4] Slosman DO, Davidson D, Brill AB, Alderson PO. Iodine-123Metaiodobenzylguanine uptake in the isolated rat lung: a potential marker of endothelial cell function. Eur J Nucl Med 1988; 13: 5437.

[5] Slosman DO, Donath A, Alderson PO. ${ }^{131}$ I-metaiodobenzylguanidine and ${ }^{125}$ I-iodoamphetamine; Parameters of lung endothelial cell function and pulmonary vascular area. Eur J Nucl Med 1989; 15: 207-10.

[6] Slosman DO, Polla BS, Donath A. ${ }^{131}$ I-MIBG pulmonary removal: a biochemical marker of minimal lung endothelial cell lesions. Eur J Nucl Med 1990; 16: 633-7.

[7] Takabatake N, Arao T, Sata M, et al. Involvement of pulmonary endothelial cell injury in the pathogenesis of pulmonary fibrosis: clinical assessment by 123I-MIBG lung scintigraphy. Eur J Nucl Med Mol Imaging 2005; 32: 221-8.

[8] Yoshiike F, Koizumi T, Urushihata K, Hanaoka M, Kubo K. Reduced lung uptake of iodine-123 metaiodobenzylguanidine in patients with MPO-ANCA positive vasculitis. Respiration 2005; 73: 191-6.

[9] Schoene RB. Pulmonary edema at high altitude; review, pathophysiology and update. Clin Chest Med 1985; 6: 3-26.

[10] Urushihata K, Koizumi T, Hanaoka M, et al. Reduced lung uptake of iodine-123-metaiodobenzyguanidine in high-altitude pulmonary edema. Respirology 2008; 13: 897-902.

[11] Fukuoka M, Yano S, Giaccone G, et al. Multi-institutional randomized phase II trial of gefitinib for previously treated patients with advanced non-small-cell lung cancer. J Clin Oncol 2003; 21: 2237-46.

[12] Lynch TJ, Bell DW, Sordella R, et al. Activating mutations in the epidermal growth factor receptor underlying responsiveness of non-small-cell lung cancer to gefitinib. N Engl J Med 2004; 350: 2129-39.

[13] Paez JG, Jänne PA, Lee JC, et al. EGFR mutations in lung cancer: correlation with clinical response to gefitinib therapy. Science 2004; 304: 1497-500.

[14] Inoue A, Saijo Y, Maemondo M, et al. Severe acute interstitial pneumonia and gefitinib. Lancet 2003; 361: 137-9.

[15] Kudoh S, Kato H, Nishiwaki Y, et al. Interstitial lung disease in Japanese patients with lung cancer: a cohort and nested casecontrol study. Am J Respir Crit Care Med 2008; 177: 1348-57.

[16] Nakagawa M, Nishimura T, Teramukai S, et al. Interstitial lung disease in gefitinib-treated Japanese patients with non-small cell lung cancer - a retrospective analysis: JMTO LC03-02. BMC Res Notes 2009; 2: 157.

[17] Modi S, Seidman AD. An update on epidermal growth factor receptor inhibitors. Curr Oncol Rep 2002; 4: 47-55.

[18] Wu W, Onn A, Isobe T, et al. Targeted therapy of orthotopic human lung cancer by combined vascular endothelial growth factor and epidermal growth factor receptor signaling blockade. Mol Cancer Ther 2007; 6: 471-83.

[19] Madtes DK, Busby HK, Strandjord TP, Clark JG. Expression of transforming growth factor-alpha and epidermal growth factor receptor is increased following bleomycin-induced lung injury in rats. Am J Respir Cell Mol Biol 1994; 11: 540-51.

[20] Ishii Y, Fujimoto S, Fukuda T. Gefitinib prevents bleomycininduced lung fibrosis in mice. Am J Respir Crit Care Med 2006; 174: 550-6. 